\title{
A New Low Reynolds Number Facility for Active Flow Control Applications
}

\author{
Matthew J. Munson; William B. Dickson† Tim Colonius; and Morteza Gharib ${ }^{\ddagger}$ \\ California Institute of Technology, Pasadena, CA, 91125, USA
}

\begin{abstract}
Recent interest in gaining understanding of the dynamics and behavior of the leadingedge vortex structure observed in biological flight systems has prompted the construction of a new low Reynolds number facility. This facility, a recirculating oil tunnel, gives several distinct advantages over similar facilities, utilizing water or air as the working fluid, for this kind of study. Additionally, as understanding is gained, active flow control strategies leading to the stabilization of the leading-edge vortex structure will be investigated, and this facility is specially equipped to enable this study. The tunnel has been designed and installed at the California Institute of Technology Graduate Aeronautical Laboratories. Design features of the facility will be discussed, along with some preliminary measurements conducted on a NACA 0012 wing.
\end{abstract}

\section{Introduction}

In pursuit of integrated closed-loop flow and flight control for stabilization and regulation of vortex formation in separated flows, a new low Reynolds number facility has been designed and installed at the Graduate Aeronautical Laboratories at California Institute of Technology.

Observations of insect flight show intriguing unsteady high-lift mechanisms that rely heavily on stabilization of leading-edge vortex (LEV) structures. A multi-disciplinary university research initiative (MURI) has been formed in order to explore the benefits of understanding and manipulating this LEV stabilization process for micro air vehicle applications. Flow control work at higher Reynolds numbers provides a strong likelihood that mass injection control strategies will be able to strongly influence vortex shedding and result in lift enhancement.

This paper discusses the design features that makes this facility uniquely suited to pursue these investigations.

\section{I.A. Background}

The motivation for this work is provided by observation of high lift mechanisms in insect flight, where Reynolds numbers are typically below $10^{4}$ and aspect ratios are less than three. For the purposes of the MURI, the chord Reynolds number is around $10^{3}$ with an aspect ratio of two. Previous studies have shown that for this aerodynamic regime, stabilization of the leading-edge vortex is one of the primary mechanisms for lift enhancement. ${ }^{1,2}$

To enable effective studies for this Reynolds numbers range, a recirculating oil tunnel was designed and installed. Flow speeds up to $25 \mathrm{~cm} / \mathrm{s}$ are currently achievable through the $50 \mathrm{~cm} \times 50 \mathrm{~cm} \times 150 \mathrm{~cm}$ test

* Graduate Student, Aeronautics Option.

${ }^{\dagger}$ Postdoctoral Scholar, Bioengineering Option.

$\ddagger$ Professor, Mechanical Engineering Option.

$\S$ Professor, Aeronautics and Bioengineering Options. 
section. Mineral oil with kinematic viscosity of $1.50\left(10^{-5}\right) \mathrm{m}^{2} / \mathrm{s}$ (nearly 13 times that of water) is the working fluid. This kinematic viscosity was chosen in order to ensure sufficient force production in this Reynolds number range, as well as keeping the model size large enough to easily implement the actuation scheme.

\section{Facility Setup}

\section{II.A. Recirculating Oil Tunnel}

The facility, as mentioned above, is a recirculating tunnel with oil as the working fluid. Figure 1 is a schematic layout of the facility. The working fluid is driven through the tunnel by a large pump (Aurora Pumps, Model $344 \mathrm{~A}$ ). The flow is provided to the supply chamber through a perforated pipe. Flow conditioning, consisting of a perforated plate, honeycomb, and several fine screens, is in place to straighten the flow and reduce the freestream turbulence levels. The flow then travels through the test section, ultimately turning through guide vanes into the large return section and then back to the pump. The tunnel is constructed from acrylic and rests on a welded steel frame. The all acrylic construction provides premium optical access for quantitative flow measurement techniques.

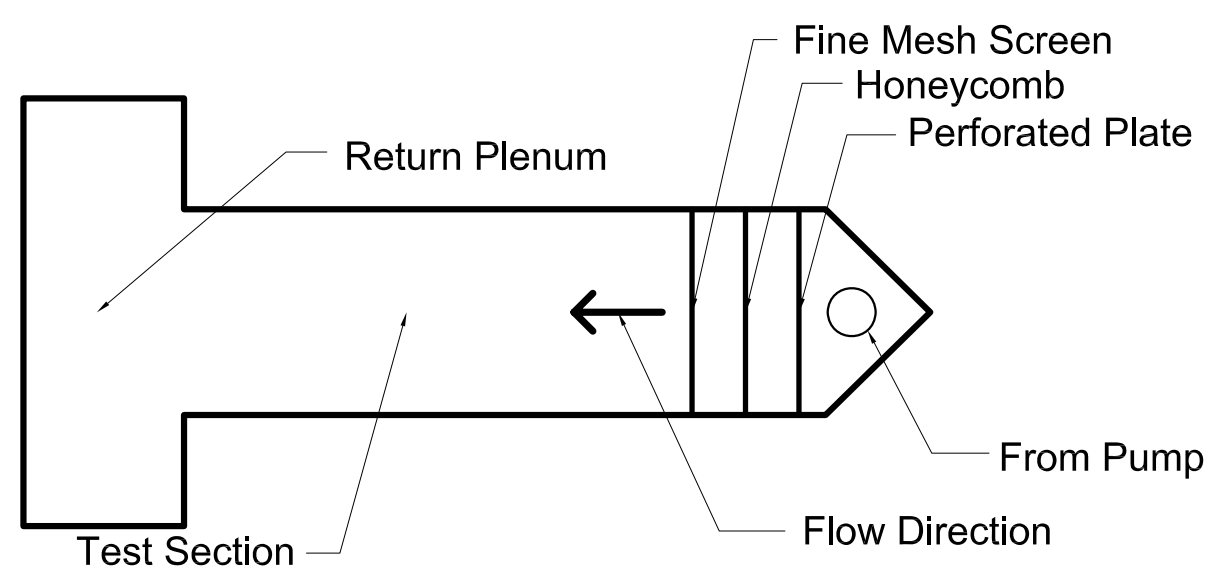

Figure 1. Oil tunnel schematic.

From the schematic, it is clear that no contraction is present in the supply side of the tunnel. This was done intentionally in order to keep the boundary layer growth from intruding on the freestream in the test section. The freestream is currently nicely uniform over approximately $80 \%$ of the test section at the model location. Also, the tunnel is designed to eliminate the free surface at the top of the test section. A series of interlocking lid slats are secured in place (with only a small opening for the model support sting) and then the test section is over-filled in order to provide a no-slip boundary condition at the top of the tunnel. Finally, two nickel-plated copper cooling loops are installed in the return section in order to provide temperature control of the tunnel, which is important to maintain stable fluid viscosity values and sensor calibrations.

Models are mounted from above the tunnel via a rigid assembly, which is suspended from the ceiling. The model sting is connected to this assembly, which provides nearly unlimited freedom in positioning the model within the tunnel, including angle of attack. Figure 2 shows the tunnel in its current configuration.

\section{II.B. Oil Characteristics}

The oil currently in use is from the Chevron Superla White Oil product line. This line consists of six odorless, colorless oils, comprised of mixtures of saturated paraffinic and naphthenic hydrocarbons, with

$$
2 \text { of } 8
$$




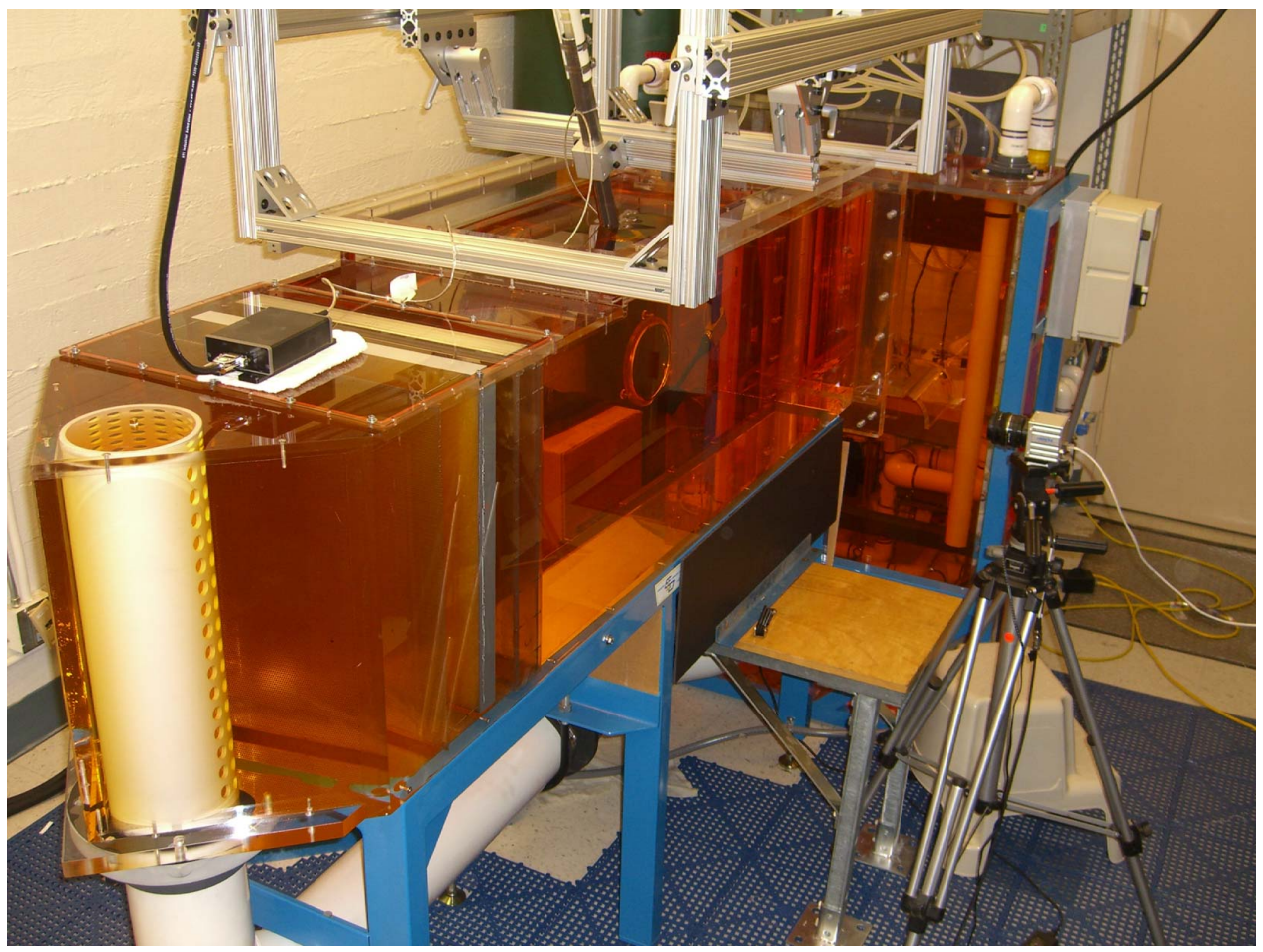

Figure 2. Low Reynolds number oil tunnel facility.

densities ranging from $831 \mathrm{~kg} / \mathrm{m}^{3}$ to $868 \mathrm{~kg} / \mathrm{m}^{3}$. Kinematic viscosities range from 7.8 to $63.7 \mathrm{cSt}$ at $40^{\circ} \mathrm{C}{ }^{6}$ These oils are miscible in one another, allowing for the possibility of fine-tuning the viscosity value of the working fluid. Additionally, the viscosity of these oils have strong temperature dependence. Figure 3 shows the dynamic viscosity of Superla White Oil 5 (currently in the facility) as a function of temperature. As is immediately obvious, control of the facility's temperature is crucial to locking in the viscosity over the course of an experiment.

The above characteristics of these oils provide the motivation for using them as the working fluid for low Reynolds number testing. Typical aerodynamic studies are more often done in air or water facilities, but the use of oil allows for generation of easily measurable forces at low Reynolds number. To provide an example, Table 1 shows the required tunnel speed and force generated on a model with characteristic chord length $(c)$ $10 \mathrm{~cm}$, aspect ratio $(A) 2$, Reynolds number $10^{3}$ and force coefficient $\left(C_{F}\right)$ of 1 . The representative force is calculated for a rectangular wing via Equation 1, where $\rho$ is the density, and $U_{\infty}$ is the freestream speed.

$$
F=\frac{1}{2} \rho U^{2} A c^{2} C_{F}
$$

As is easily seen, the forces generated in the oil tunnel are much larger (and therefore easier to measure) in an experiment than those in a water or wind tunnel. Additionally, the tunnel speed would have to be very small for a similarly sized water facility, which is difficult to control. Thus, the oil provides a distinct advantage for this kind of work.

\section{II.C. Flow Control Architecture}

As mentioned in the Introduction, the goal of this tunnel is to facilitate the study of flow control methodologies for low Reynolds number fliers. To that end, a significant effort has been made to provide the requisite 


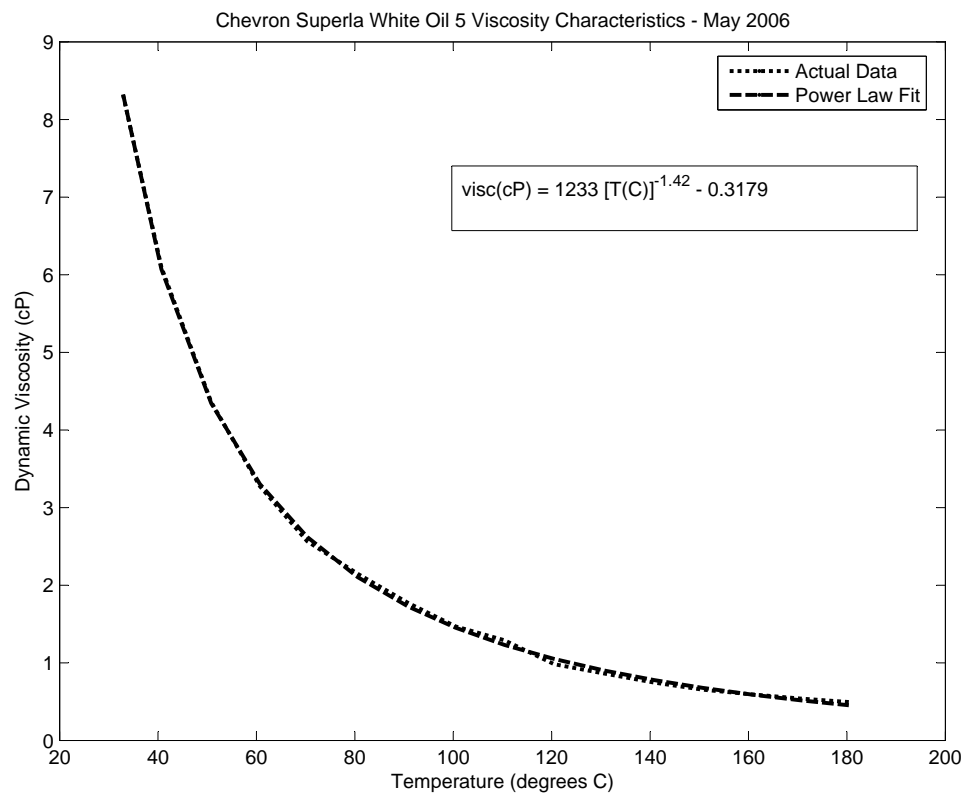

Figure 3. Chevron Superla White Oil 5 viscosity vs. temperature.

Table 1. Freestream speeds $(U)$ and force $(F)$ generated on a $10 \mathrm{~cm}$ model at $\operatorname{Re}=1000$ and $C_{F}=1$

\begin{tabular}{rrr} 
Fluid & $U(\mathrm{~cm} / \mathrm{s})$ & $F(\mathrm{~N})$ \\
\hline Water & 1 & $1.1\left(10^{-3}\right)$ \\
Air & 18 & $3.9\left(10^{-4}\right)$ \\
Oil & 15 & $1.9\left(10^{-1}\right)$
\end{tabular}

infrastructure. In these initial studies, manipulation of the flow physics around the model will be attempted via steady and unsteady injection of mass and momentum through the surface of the model.

The current infrastructure for a single channel is shown schematically in Figure 4. Supply for the actuation is provided by an auxiliary pump, which draws fluid from the return section of the tunnel. The auxiliary pump is currently capable of providing approximately 25 psi to the actuation system manifold, downstream of which the flow rate and actuation frequency for each channel are regulated before being sent to the model. Frequencies up to approximately $15 \mathrm{~Hz}$ are generated by solenoid valves, which are digitally controlled from a PC. Mass flow rate will eventually be converted to computer control, but is currently set by hand. Flow rates through each actuator channel is on the order of $10 \mathrm{~mL} / \mathrm{s}$, which yields a momentum coefficient $C_{\mu}=\left[\rho_{a c t} U_{a c t}^{2} A_{a c t}\right] /\left[(1 / 2) \rho_{\infty} U_{\infty}^{2} S\right]=0.002$, which matches well with requirements of other flow control applications.

\section{II.D. Measurement Capabilities}

In pursuit of understanding the interaction between the leading edge vortex and the resultant forces generated on an airfoil, both flow field quantities and forces will be measured.

Quantitative flow visualization will be achieved with traditional two-component digital particle image velocimetry (DPIV), ${ }^{3}$ as well as a new implementation of a fully three-dimensional technique, defocused digital particle image velocimetry (DDPIV). ${ }^{4}$ Use of DDPIV allows the three-dimensional structure of the vortex 


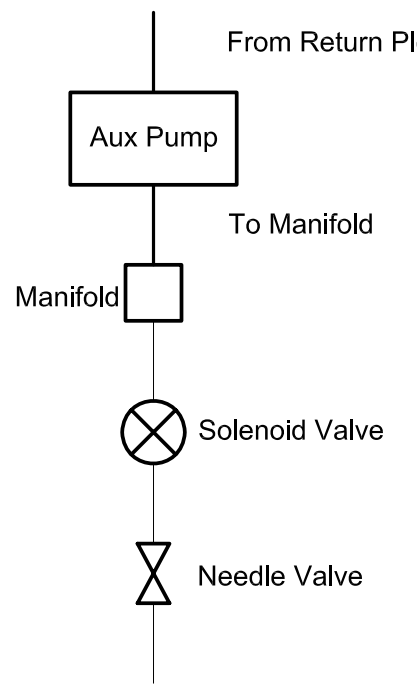

Actuator on Model

Figure 4. Actuator infrastructure (one channel).

formation to be measured directly, rather than inferred from successive two-dimensional slices. Additionally, DDPIV allows for quantification of the uncertainty existent in the DPIV method. This is crucial in order to implement DPIV as a real-time sensor for closed loop flow control experiments. The oil is seeded with silver coated glass spheres (47 $\mu \mathrm{m}$ nominal diameter) as tracer particles. A filter unit in the auxiliary flow handling unit allows for the extraction of the particles in order to select another particle size if desired. A $200 \mathrm{~mJ}$ pulsed Nd:Yag laser provides the illumination source, with appropriate optics in place to provide a two-dimensional sheet (DPIV) or three-dimensional cylinder (DDPIV). Images are captured to a single CCD array for DPIV and to three independent CCD arrays (integrated into a camera system) for DDPIV.

Forces and moments are measured using a six-component force balance (ATI Nano-43). The force balance is capable of measuring forces up to $18 \mathrm{~N}$ and torques up to $250 \mathrm{~N}-\mathrm{mm}$. The sensor employs silicon strain gauges and has a center hole, which allows the actuator plumbing to pass through the sensor. A data acquisition system captures the deflection of the strain gauges and converts them via calibration to lift and drag forces.

\section{Airfoil Models}

The airfoil profile chosen for this experiment is a NACA 0012, chosen primarily to provide sufficient room for the actuator plumbing to be fully contained within the model. The models also all have an aspect ratio of 2. The models are constructed as a single piece from ABS plastic using a rapid prototyping method. Actuators, in the form of slots located on the surface of the airfoil attempt to manipulate the local flow dynamics about the wing, with the goal of gaining control authority over the vortex formation process.

All current models contain seven actuation slots. Table 2 shows the placement location and direction of the actuators, as well as actuator slot size. A sting without a wing attached has also been constructed to allow for an estimate of the forces due to the sting to be made.

The actuators consist of seven slots with width $0.5 \mathrm{~mm}$ and length $20 \mathrm{~mm}$. Five are placed along the leading edge and one on each of the tips. Flow out of the leading edge actuators is directed downstream and flow out of the tip actuators is directed inboard. Flow rates through each actuator is on the order of $10 \mathrm{~mL} / \mathrm{s}$, which yields a momentum coefficient $C_{\mu}=\left[\rho_{\text {act }} U_{\text {act }}^{2} A_{\text {act }}\right] /\left[(1 / 2) \rho_{\infty} U_{\infty}^{2} S\right]=0.002$, which matches 
Table 2. Airfoil models. All are NACA 0012 profiles except for semi-circular, which is a flat plate.

\begin{tabular}{rrrrr} 
Planform & Slot Location & Direction & Slot Width $(\mathrm{mm})$ & Slot Length $(\mathrm{mm})$ \\
\hline Rectangular & Leading Edge $(5)$ \& Tip $(2)$ & Downstream & 0.5 & 20 \\
Rectangular & Leading Edge & Upstream & 0.5 & 25 \\
Rectangular & Mid-chord & Downstream & 0.5 & 20 \\
Semi-circular & Leading Edge & Upstream & 0.5 & 35
\end{tabular}

well with requirements of other flow control applications.

A representative airfoil and sting are shown in Figure 5. The view is towards the underside of the wing and the actuators are colored for easy identification. The back of the sting mounts directly to the face of the force balance sensor.

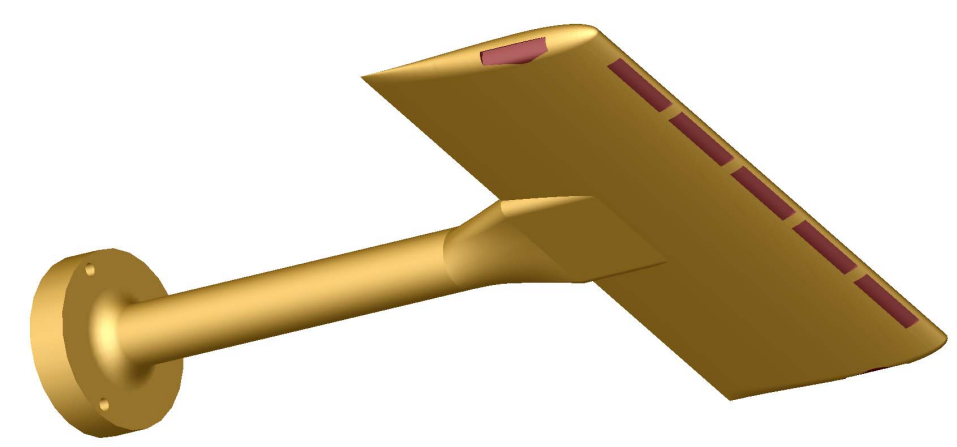

Figure 5. NACA 0012 airfoil with flow control actuators (colored sections along leading edge and tips).

\section{Preliminary Results}

Representative lift-drag polars for the wing shown in Figure 5 at Reynolds number 1150 are shown in Figure 6. Corrections for gravitational effects have been made, but the effect of the sting has not been accounted for, so the drag measurements are higher than expected.

A representative DPIV image sequence at Reynolds number 700 is shown in Figure 7. Here, the model is located near the center of the image and the low speed wake can be clearly seen. The colorbar in the top left shows the speed ranging between 0 and $0.15 \mathrm{~m} / \mathrm{s}$. The model is at $45^{\circ}$ angle of attack and is actually "upside-down" in this image. The arrow indicates the velocity center of a shed vortex structure, which moves approximately $1.2 \mathrm{~cm}$ from the top left image (a) to the bottom right image (d). Based on analysis of a more extensive sequence, the shedding frequency is calculated to be around $1 \mathrm{~Hz}$.

6 of 8 


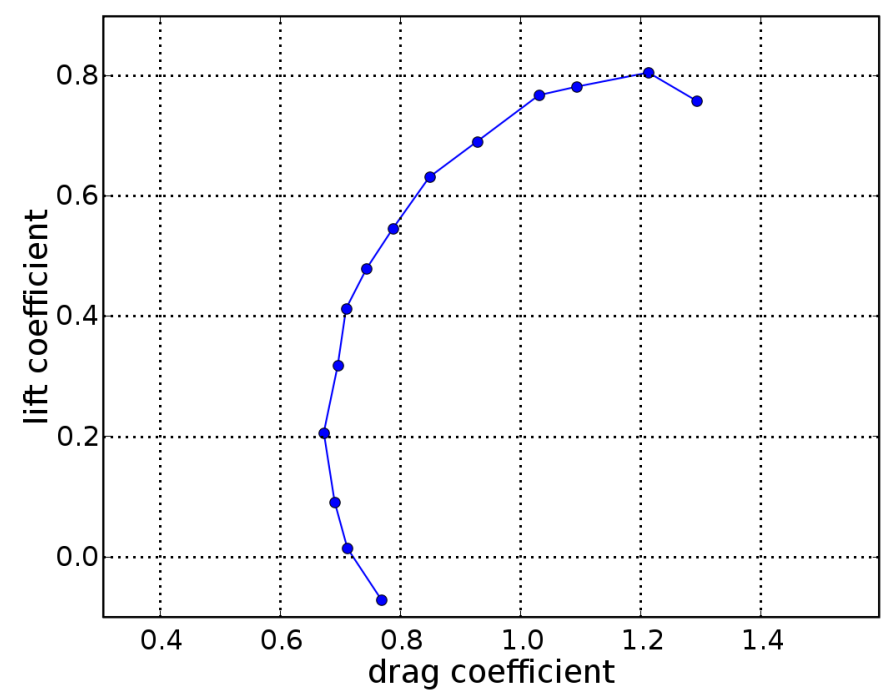

Figure 6. Baseline lift-drag polars for NACA 0012 model.

\section{Conclusions}

A new facility has been constructed in pursuit of low Reynolds number aerodynamic studies. In addition, the facility is uniquely suited to investigate flow control strategies. A companion paper ${ }^{5}$ presents computational results for low Reynolds number, low aspect-ratio airfoils, including actuator models.

\section{Acknowledgements}

This effort supported by a Multidisciplinary Research Initiative from the United States Air Force Office of Scientific Research (FA9550-05-1-0369, Program Manager: Dr. Fariba Fahroo).

\section{References}

${ }^{1}$ C.P. Ellingtion, C. van den Berg, A.P. Willmott, and A.L.R. Thomas, "Leading-edge Vortices in Insect Flight," Nature, Vol. 384, pp. 626-630, 1996.

${ }^{2}$ J.M. Birch, W.B. Dickson, and M.H. Dickinson, "Force Production and Flow Structure of the Leading-Edge Vortex on Flapping Wings at High and Low Reynolds Numbers," Journal of Experimental Biology, Vol. 207, pp. 1063-1072, 2004.

${ }^{3}$ C.E. Willert and M. Gharib, "Digital Particle Image Velocimetry," Experiments in Fluids, Vol. 10, pp. 181-193, 1991.

${ }^{4}$ F. Pereira and M. Gharib, "Defocusing digital particle image velocimetry and the three-dimensional characterization of two-phase flows," Measurement Science Technology, Vol. 13, pp. 683694, 2002.

${ }^{5}$ K. Taira, W.T. Joe, T. Colonius and D.G. MacMynowski, "Three-Dimensional Simulation of Flow Control Applied to a Low-Aspect-Ratio Airfoil," AIAA Conference Paper, to be submitted, 2008.

${ }^{6}$ Chevron Products Company. Chevron Superla White Oils. Data Sheet WO-38. (located at https://www.cbest.chevron.com/generated/MSDS/PDS7677732.PDF at time of writing.) March 2007.

7 of 8 


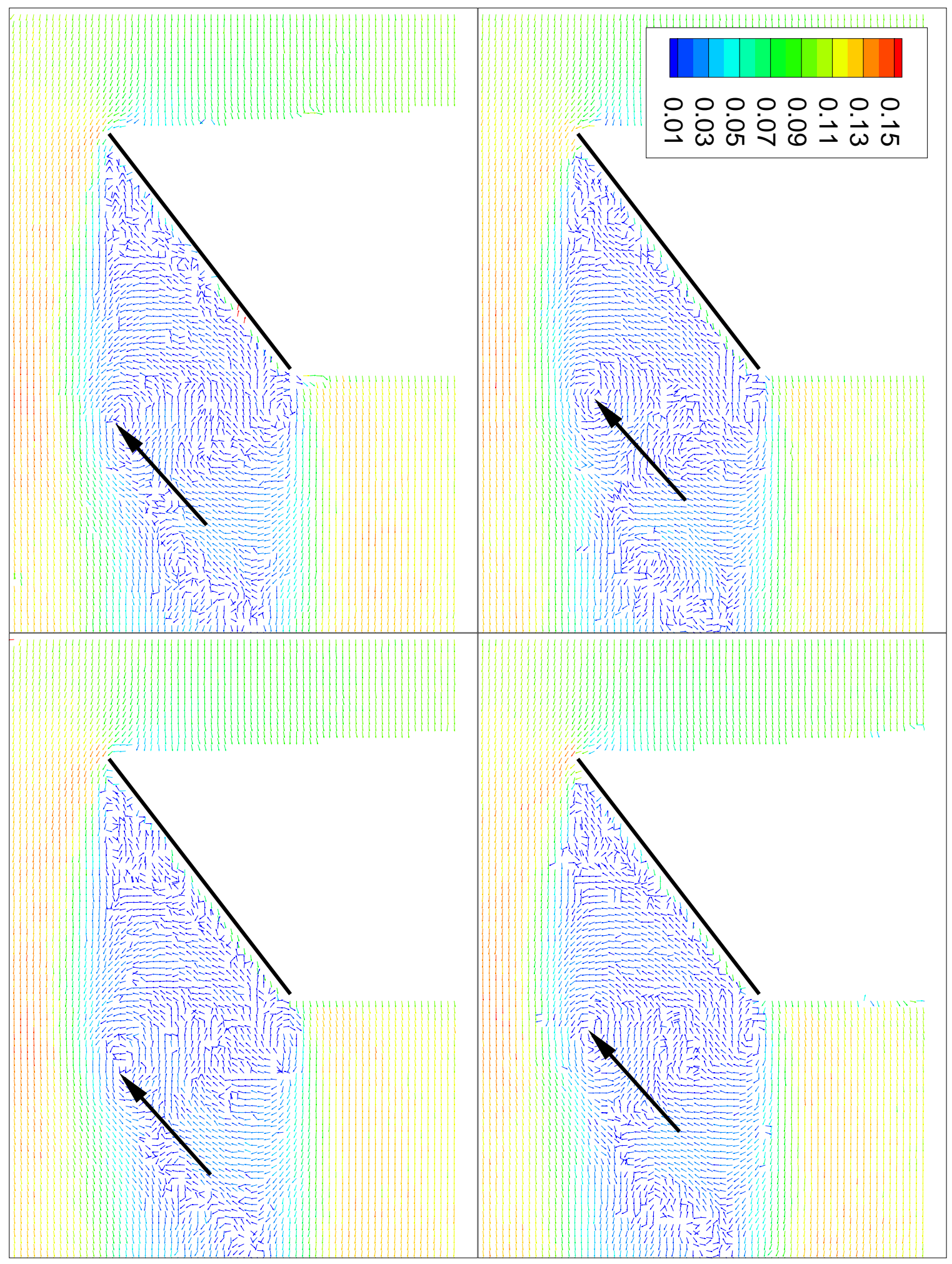

Figure 7. Representative PIV image sequence.

$$
8 \text { of } 8
$$

